\title{
Mapeamento da Fragilidade Ambiental na Bacia do Rio Aldeia Velha, RJ
}

\author{
Ivana Cola Valle ${ }^{1}$, Márcio Rocha Francelino ${ }^{2}$, Helena Saraiva Koenow Pinheiro ${ }^{3}$ \\ ${ }^{1}$ Departamento de Ciências Biológicas, Universidade Estadual de Santa Cruz - UESC, Ilhéus/BA, Brasil \\ ${ }^{2}$ Departamento de Silvicultura, Universidade Federal Rural do Rio de Janeiro - UFRRJ, Seropédica/RJ, Brasil \\ ${ }^{3}$ Departamento de Ciências do Solo, Universidade Federal Rural do Rio de Janeiro - UFRRJ, Seropédica/RJ, Brasil
}

\begin{abstract}
RESUMO
Este trabalho apresenta um modelo para determinar a fragilidade ambiental em bacias hidrográficas. O estudo foi realizado na Bacia do Rio Aldeia Velha, RJ, localizada na zona de contato e transição entre a baixada litorânea e o relevo montanhoso da Serra do Mar. Fatores que influenciam a ocorrência de processos erosivos foram integrados por algoritmos em um SIG para construção de classes de fragilidade. A análise multicriterial considerou o modelo numérico de terreno, dados oficiais sobre variáveis ambientais, imagem orbital de alta resolução e a opinião de especialistas. Através de informações secundárias sobre pedologia, intensidade das chuvas e declividade do terreno gerou-se o Mapa de Fragilidade Potencial (MFP). Através da combinação desse mapa com informações sobre uso e cobertura da terra obteve-se o Mapa da Fragilidade Emergente (MFE). Os resultados mostram que mais de $70 \%$ da área da bacia possui fragilidade ambiental considerada alta ou muito alta, tanto potencial como emergente. Os Processos Erosivos Aparentes (PEA) relacionaram-se positivamente com as áreas de alta fragilidade nos produtos cartográficos finais, destacando regiões mais propensas à intensificação de movimentos de massa e prioritárias para prevenção contra perda de solo. Os modelos geraram informações importantes para o planejamento territorial, possibilitando um zoneamento acessível e de fácil atualização para as prefeituras municipais e organizações da sociedade civil, inclusive para o monitoramento das áreas de alta fragilidade ambiental.
\end{abstract}

Palavras-chave: geoprocessamento, diagnóstico, planejamento territorial.

\section{Mapping of Environmental Fragility in the Aldeia Velha River Basin, State of Rio de Janeiro, Brazil}

\begin{abstract}
This paper presents an approach to mapping the environmental fragility of river basins. The study was performed at the Aldeia Velha river basin, a rainforest watershed located between Rio de Janeiro's coastal plains and the Serra do Mar highlands. A multiple-criteria analysis was performed involving factors that affect the risk of erosion; these variables were analyze using GIS tools and were integrated by algorithm in order to form a description of the different classes of environmental fragility in the basin. The multicriteria analysis considered the use of a numerical land model, official data, orbital imagery and the opinion of subject-matter experts. A Map of Potential Fragility (MPF) was generated through the collection of secondary information such as soil types, rainfall intensity and terrain slope. This initial map was later combined with a land-use projection to produce a Map of Emerging Fragility (MEF). The mapping results pointed to a highly fragile environment, where more than 70 percent of the basin's area was classified with high or very high degree of fragility, in both the potential and emerging context. The Apparent
\end{abstract}


Erosion Processes (AEP) were positively correlated in the areas with a higher level of fragility in the final map products, as well as highlighted the locations most prone to the intensification of erosion processes. The models generated important information for land-use planning, enabling affordable and easy upgrade for municipal governments and civil society organizations to monitor high fragility areas.

Keywords: geoprocessing, diagnosis, territorial planning.

\section{INTRODUÇÃO}

A análise ambiental e o planejamento territorial têm como base as condições da paisagem e as características naturais da região, considerando principalmente informações como declividade, pedologia, intensidade pluviométrica, uso da terra e cobertura vegetal, de forma a viabilizar um efetivo gerenciamento ambiental (Bojórquez-Tapia et al., 2013; Martín-Duque et al., 2012; Ross, 2012). A aplicação de geotecnologia para a elaboração de produtos cartográficos relacionados com a fragilidade do ambiente difunde-se cada vez mais no Brasil, uma vez que configura conjunto de ferramentas altamente eficientes para gestão territorial e promoção da qualidade da paisagem (Rodrigues et al., 2001; Francisco et al., 2008). As ferramentas que compõem os Sistemas de Informação Geográfica (SIGs) facilitam a realização de operações complexas, permitindo a integração de grande volume de dados de natureza e escalas diferentes como, por exemplo, imagens de satélite, cartas topográficas e informações pontuais.

Fragilidade ambiental é uma medida da sensibilidade intrínseca dos ecossistemas às pressões ambientais, associadas também a quaisquer ameaças que sejam capazes de perturbar o equilíbrio de um sistema (Ratcliffe, 1971). Os sistemas ambientais podem responder de diferentes maneiras às intervenções humanas nos componentes da paisagem, como relevo, solo, clima, recursos hídricos e cobertura vegetal. Mapear a fragilidade ambiental permite definir áreas mais frágeis e que merecem maior atenção, pois sua má utilização pode resultar no comprometimento de todo sistema. O grau de um impacto sobre o equilíbrio de um sistema, dependendo do tipo de intervenção, pode ser maior ou menor em função das características intrínsecas do ambiente, ou seja, de sua fragilidade ambiental (Franco et al., 2013).

O mapa de fragilidade ambiental constitui importante ferramenta utilizada pelos órgãos públicos para o planejamento territorial, em escala regional ou local.
Ele pode ser utilizado por pequenos proprietários e produtores rurais, uma vez que possibilita a avaliação das características ambientais de forma integrada, considerando suas potencialidades e restrições (Kawakubo et al., 2005; Tomczyk, 2011). Para ser efetivo, o planejamento das formas de uso e ocupação da terra deve considerar a fragilidade do ambiente à degradação, monitorando processos que afetam diretamente o fornecimento de bens e serviços ambientais, como características relacionadas à erosão do solo, assoreamento de cursos d'água e perda de biodiversidade.

A avaliação sistêmica de um ambiente constitui base para o estudo integrado da relação entre os seus elementos naturais, possibilitando um gerenciamento voltado à conservação da paisagem e biodiversidade (Spörl \& Ross, 2004). A identificação de unidades naturais e dos graus de fragilidade (potencial e emergente) da paisagem proporcionam melhores definições para a etapa de planejamento territorial, base para traçar diretrizes e propor ações de zoneamento e gestão das áreas especiais, que necessitam de maiores esforços para conservação do equilíbrio ambiental (MartínDuque et al., 2012; Tomczyk, 2011).

Através da inter-relação entre as variáveis ambientais (relevo, tipo de solo, precipitação e uso da terra), modelos computacionais aplicados ao estudo da fragilidade para fins de ordenamento territorial resultam em mapeamentos temáticos, nos quais a área de estudo é classificada segundo uma escala de fragilidade ambiental (Jain \& Goel, 2002). Nessa perspectiva, modelos qualitativos empíricos são empregados na análise da fragilidade ambiental para avaliação do fenômeno da erosão, no que diz respeito a sua origem, evolução e condicionantes, podendo fundamentar-se na experiência de especialistas (Kappes et al., 2012; Cardinali et al., 2002).

Baseado na concepção ecológica das Unidades Ecodinâmicas (Tricart, 1977), o ambiente é analisado sob o prisma da Teoria de Sistemas, que parte do pressuposto que na natureza as trocas de energia e 
matéria ocorrem de acordo com o equilíbrio dinâmico entre os componentes da paisagem. Todavia, esse equilíbrio pode ser alterado pelas intervenções humanas nos diversos sistemas da natureza, que podem gerar desequilíbrios, levando a impactos temporários ou até mesmo irreversíveis. Nesse sentido é necessário que esses sistemas sejam avaliados de forma integrada para análise da fragilidade ambiental, baseando-se na funcionalidade intrínseca entre as componentes físicas, as bióticas e as atividades humanas.

Ross (1994) propôs um método de análise empírica na determinação da fragilidade de ambientes naturais e antropizados, tendo como fundamento o conceito das Unidades Ecodinâmicas (Tricart, 1977). Esse método de planejamento territorial sintetiza diferentes padrões de fragilidade em Unidades Ecodinâmicas Estáveis (Instabilidade Potencial) e Unidades Ecodinâmicas Instáveis (Instabilidade Emergente), no qual o Mapa de Fragilidade Potencial representa fatores intrínsecos à paisagem e o Mapa de Fragilidade Emergente considera também a cobertura da terra e intervenções humanas.

Outro método baseado no conceito de Ecodinâmica de Tricart (1977) foi apresentado por Crepani et al. (2001) para representar a vulnerabilidade natural à perda de solo através da reinterpretação de mapas temáticos e de imagens de satélite. Nesse método, a vulnerabilidade das unidades de paisagem é estabelecida com base na relação morfogênese/pedogênese, por meio de uma escala de valores relativos e empíricos para cada plano de informação (PI) considerado.

Em relação aos métodos de cálculo da fragilidade, o modelo de Crepani et al. (2001) trabalha com valores aritméticos médios para o produto final, no qual todas as variáveis têm a mesma importância (peso) para o estabelecimento dos graus de fragilidade das Unidades Territoriais Básicas (UTB). Em contrapartida, o modelo proposto por Ross (1994) adota o agrupamento dos índices das variáveis e considera o relevo (mapa de declividade) como variável principal, com uma importância maior na definição dos graus de fragilidade.

O presente trabalho teve como objetivo elaborar um modelo para o diagnóstico da fragilidade ambiental em bacias hidrográficas. Para isso, foram analisados fatores que representam qualitativamente riscos de degradação do ambiente, especialmente em relação ao desencadeamento de processos erosivos.
Configurou-se um método adaptado de resposta de bacias hidrográficas à erosão, sendo gerados produtos que, integrados, representaram um instrumento relativamente acessível para planejamento territorial em escala local, ambientalmente conservacionista e economicamente viável. Assim oferecemos subsídios para gestão e monitoramento de bacias hidrográficas, com especial atenção para as formas de ocupação da terra em áreas de risco, isto é, de alta fragilidade e mais propensas à erosão.

\section{MATERIAL E MÉTODOS}

\subsection{Caracterização da área em estudo}

A bacia hidrográfica do Rio Aldeia Velha está situada na zona costeira, ou região das baixadas litorâneas, na bacia hidrográfica do Rio São João, no estado do Rio de Janeiro (Figura 1), entre os paralelos $22^{\circ} 23^{\prime}$ $22^{\circ} 33^{\prime} \mathrm{S}$ e os meridianos $42^{\circ} 15^{\prime}-42^{\circ} 19^{\prime} \mathrm{W}$, possuindo área de drenagem de aproximadamente 14.400 ha. O Rio Aldeia Velha, um dos principais afluentes pela margem esquerda do Rio São João, é limite físico e político entre os municípios de Silva Jardim e Casimiro de Abreu e responde pelo abastecimento de água de 10 municípios da Região dos Lagos, uma das de maior potencial turístico do estado do Rio de Janeiro.

A bacia hidrográfica em estudo está totalmente inserida na Área de Proteção Ambiental (APA) da Bacia do Rio São João, abrangendo aproximadamente 56\% da Reserva Biológica (REBIO) Poço das Antas, parte de seu entorno e Zona de Amortecimento. A área encontra-se sob o domínio da Mata Atlântica e constitui hábitat de espécies endêmicas da fauna brasileira como, por exemplo, o Mico-leão-dourado (Leontopithecus rosalia), a Preguiça-de-coleira (Bradypus torquatus) e a Borboleta-da-praia (Parides ascanius) (Brasil, 2005).

O clima local é quente e úmido, com uma estação seca no inverno, sendo classificado como Aw, segundo Köppen (1948). De maneira geral, a região das baixadas litorâneas no estado do Rio de Janeiro, apresenta grande variação pluviométrica, com menor pluviosidade no litoral próximo à região de Arraial do Cabo e um aumento gradativo à medida que se avança para o interior, alcançando máximos valores nas proximidades da Região Serrana (Montebeller et al., 2007). 


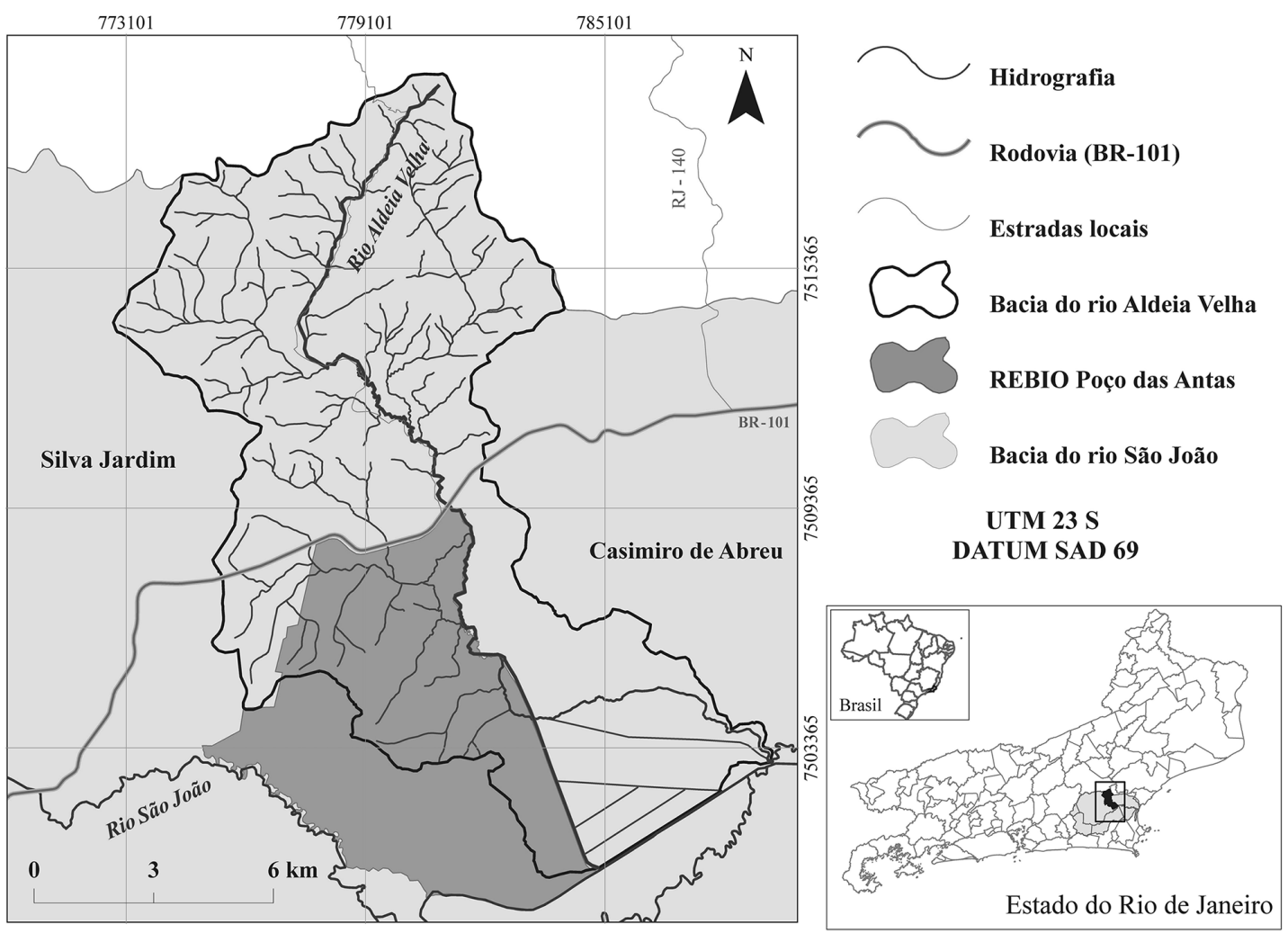

Figura 1. Localização da área em estudo.

Figure 1. Location of study área.

\subsection{Atributos do terreno e definição dos critérios}

Para a obtenção dos mapas finais foram gerados diversos mapas intermediários. Os atributos utilizados como critério para definição das classes de fragilidade ambiental foram os seguintes temas ou planos de informação (PI): precipitação média anual (mm/ano), declividade do terreno (\%), classes de solo (Embrapa, 2006) e uso/cobertura da terra (imagem de satélite Ikonos). Os mapas originais foram discretizados através de reclassificação com base em limites pré-definidos e hierarquizados, de forma a permitir a sobreposição e os cálculos entre os distintos PI utilizados no diagnóstico da fragilidade ambiental. Todas as análises foram realizadas no software ArcGIS Desktop v.10 ${ }^{\circledR}$ (ESRI, 2010).

Com o objetivo de aumentar a consistência entre os mapas utilizados e ajustar a escala de origem para a escala do trabalho (Spörl, 2007; Crepani et al., 2001), efetuou-se a reinterpretação dos mapas preexistentes utilizando-se como "âncora" a imagem de satélite Ikonos (no ano de 2006) e dados georreferenciados das curvas de nível, pontos cotados e traçados dos canais de drenagem na bacia em análise (IBGE, 2010). Assim, a resolução espacial adotada neste estudo foi de 10 metros, considerada adequada à escala de trabalho, resolução do MDEHC utilizado.

Em relação aos dados climáticos, especialmente distribuição e intensidade da chuva, que têm ação direta sobre a dinâmica de superfície principalmente no que tange ao intemperismo de rochas e solos, a bacia hidrográfica do Rio Aldeia Velha encontra-se na interface entre a baixada litorânea e as encostas da Serra do Mar. apresentando isoietas de precipitação média anual que variam de $2.500 \mathrm{~mm}$ na região montanhosa (alto curso do Rio Aldeia Velha) a $2.000 \mathrm{~mm}$ nas colinas (médio curso) e a $1.700 \mathrm{~mm}$ na baixada aluvial, à jusante na bacia (Brasil, 2005).

Para a classificação do mapa de precipitação utilizou-se como critério os níveis de interferência na estabilidade do ambiente de acordo com Ross (2012). A situação pluviométrica da bacia em estudo se caracteriza pela distribuição anual desigual, com período seco de 3 e 6 meses e alta concentração das chuvas no verão 
entre novembro e abril, quando ocorrem cerca de $70 \%$ a $80 \%$ do total das chuvas (Tabela 1), conforme pode ser verificado no Plano de Manejo da REBIO Poço das Antas (Brasil, 2005). Dessa forma, foi atribuída vulnerabilidade Forte - pontuação equivalente a 4 - para toda a área da bacia.

A alta pontuação conferida ao tema precipitação para toda a área de estudo é compatível com a condição natural da região, um ambiente geomorfologicamente dominado por colinas suaves, planícies de inundação (aluviais) e níveis escalonados da Serra do Mar. As características climáticas da região estão associadas à topografia acidentada e de direção concordante com a linha litorânea, que propicia a ocorrência de chuvas orográficas principalmente durante o verão. Assim, para a representação cartográfica desse atributo foi gerado um mapa digital em formato matricial de valor constante igual a 4, correspondente à classe de vulnerabilidade Forte, associada às características pluviométricas na área da bacia.

Outro atributo de suma importância para a análise da fragilidade ambiental é a declividade do terreno, que tem influência direta nos processos que condicionam a velocidade de transformação da energia potencial das águas pluviais em energia cinética e, por consequência, na intensidade dos processos erosivos (Crepani et al., 2001). Com os arquivos vetoriais das curvas de nível e hidrografia (IBGE, 2010), na escala 1:50.000, foi gerado um modelo digital de elevação hidrologicamente consistente (MDEHC) utilizando-se o algoritmo topo to raster (ESRI, 2010).

A partir do MDEHC foi elaborado o modelo da declividade do terreno, com a ferramenta Slope (ESRI,
2010), que calcula o valor máximo de mudança de cota a partir da célula central para as suas vizinhas. $O$ valor da inclinação do plano é calculado utilizando-se a técnica da máxima média (Burrough \& McDonnell, 1998), ou seja, quanto menor o valor do declive, mais plano é o terreno; quanto mais alto o valor da inclinação, mais íngreme.

Com o mapa da declividade do terreno em formato numérico contínuo foi realizada uma discretização por meio da ferramenta Reclassify (ESRI, 2010), reclassificando-se o terreno com base nos critérios estabelecidos pela Embrapa (2006), qualificando-se condições de declividade e definindo-se formas topográficas. Essas distinções foram empregadas para prover informações sobre susceptibilidade à erosão em vários ambientes e são amplamente aplicadas em várias regiões do Brasil (IBGE, 2007).

A Tabela 2 apresenta os critérios adotados para hierarquização da vulnerabilidade das classes de declividade e da área da bacia que ocupam.

Em relação à vulnerabilidade dos solos aos processos erosivos foi utilizado um mapa pedológico elaborado com base na Carta de Reconhecimento de Baixa Intensidade dos Solos no Estado do Rio de Janeiro, em escala 1:250.000 (Carvalho et al., 2003). O mapa original foi digitalizado, georreferenciado e, posteriormente, as unidades de mapeamento relativas à área em estudo foram vetorizadas.

Para facilitar a interpretação do mapa pedológico foi efetuada a reclassificação com base nos critérios propostos por Crepani et al. (2001), nos quais são designados pesos em função da erodibilidade natural das diferentes classes de solo (Tabela 3).

Tabela 1. Classes de fragilidade da variável pluviosidade: níveis hierárquicos das variações Ross (2012).

Table 1. Classes of fragilities by rainfall variable: hierarchical levels of rainfall variations Ross (2012).

\begin{tabular}{|c|c|}
\hline Níveis hierárquicos & Características pluviométricas \\
\hline 1 - Muito fraco & $\begin{array}{l}\text { Situação pluviométrica regular ao longo do ano, com volumes anuais não muito superiores a } \\
1.000 \mathrm{~mm} / \mathrm{ano} ;\end{array}$ \\
\hline 2 - Fraco & $\begin{array}{l}\text { Situação pluviométrica com distribuição regular ao longo do ano, com volumes anuais não } \\
\text { muito superiores a } 2.000 \mathrm{~mm} / \mathrm{ano} \text {; }\end{array}$ \\
\hline 3 - Médio & $\begin{array}{l}\text { Situação pluviométrica com distribuição anual desigual, com períodos secos entre } 2 \text { e } 3 \text { meses } \\
\text { no inverno e, no verão, com maior intensidade de dezembro a março; }\end{array}$ \\
\hline 4 - Forte & $\begin{array}{l}\text { Situação pluviométrica com distribuição anual desigual, com período seco entre } 3 \text { e } 6 \text { meses, e } \\
\text { alta concentração das chuvas no verão entre novembro e abril, quando ocorrem } 70 \% \text { a } 80 \% \text { do } \\
\text { total de chuvas; }\end{array}$ \\
\hline 5 - Muito forte & $\begin{array}{l}\text { Situação pluviométrica com distribuição regular, ou não, ao longo do ano, com grandes } \\
\text { volumes anuais ultrapassando } 2.500 \mathrm{~mm} / \mathrm{ano} \text {; ou, ainda, comportamento pluviométrico } \\
\text { irregular ao longo do ano, com episódios de chuvas de alta intensidade e volumes anuais } \\
\text { baixos, geralmente abaixo de } 900 \mathrm{~mm} \text { /ano (semiárido). }\end{array}$ \\
\hline
\end{tabular}


Tabela 2. Classificação da declividade na Bacia do Rio Aldeia Velha.

Table 2. Slope classification in the AldeiaVelha watershed.

\begin{tabular}{|cccc|}
\hline Declividade (\%) & Descrição do relevo & Pontuação & Área (\%) \\
\hline $0-3$ & Plano & 1 & 23 \\
\hline $3-8$ & Suave ondulado & 2 & 14 \\
\hline $8-20$ & Ondulado & 3 & 13 \\
\hline $20-45$ & Forte ondulado & 4 & 26 \\
$>45$ & Montanhoso e escarpado & 5 & 24 \\
\hline Total & & & $\mathbf{1 0 0}$ \\
\hline
\end{tabular}

Tabela 3. Valores de vulnerabilidade atribuídos às unidades de mapeamento.

Table 3. Vulnerability values of soil units.

\begin{tabular}{clcc}
\hline Sigla & \multicolumn{1}{c}{ Descrição } & Pontuação & Área (\%) \\
\hline RLd & NEOSSOLO LITÓLICO Tb Distrófico & 5 & 8 \\
\hline CXbd8 & CAMBISSOLO HÁPLICOS Tb Distrófico & 4 & 37 \\
\hline GXbd1 & GLEISSOLO HÁPLICO Tb Distrófico & 5 & 7 \\
\hline GMa & GLEISSOLO MELÂNICO Alumínico & 5 & 24 \\
\hline RUbd2 & NEOSSOLO FLÚVICO Tb Distrófico & 5 & 6 \\
\hline LVAd9 & LATOSSOLO VERMELHO-AMARELO Distrófico & 1 & 4 \\
\hline LVAd10 & LATOSSOLO VERMELHO-AMARELO Distrófico & 1 & 5 \\
\hline LVAd19 & LATOSSOLO VERMELHO-AMARELO Distrófico & 1 & 9 \\
\hline
\end{tabular}

O mapa de uso/cobertura da terra foi obtido a partir da classificação de imagem do satélite Ikonos, com resolução espacial de $1 \mathrm{~m}$, referente ao ano 2006, cedida pela Prefeitura Municipal de Silva Jardim, RJ, na escala 1:10.000 (Valle et al., 2013). Posteriormente, a classificação foi validada em campo, durante campanhas em 2007 e 2010. A Tabela 4 apresenta a classificação do mapa de uso e cobertura da terra, de acordo com os critérios propostos por Ross (1994) e Crepani et al. (2001), e a área relativa ocupada por cada classe de uso observada.

A Figura 2 apresenta os mapas dos atributos considerados no diagnóstico da fragilidade ambiental. O mapa de precipitação não foi representado por possuir um valor de superfície contínua para toda a área de estudo, pontuação 4.

A reclassificação dos produtos intermediários foi realizada de acordo com critérios hierárquicos para cada Plano de Informação (PI) - mapas temáticos em formato vetorial - e posterior conversão para grades regulares (formato raster). Foi utilizada a ferramenta Conversion tools (ESRI, 2010). Esse procedimento permitiu a integração de todos atributos do terreno. Todos produtos foram gerados com a projeção Universal Transversa de Mercator (UTM), datum horizontal SAD-69. Efetuou-se o ajuste dos mapas temáticos com base em dados georreferenciados do IBGE (2010) e
Tabela 4. Valores de vulnerabilidade atribuídos às classes de uso e cobertura da terra.

Table 4. Vulnerability values of land use classes'.

\begin{tabular}{|lcc|}
\hline \multicolumn{1}{c}{ Classes } & Pontuação & Área (\%) \\
\hline Afloramento rochoso & 5 & $<1$ \\
\hline Área Agrícola & 4 & 6 \\
Rodovia (BR-101) & 3 & $<1$ \\
\hline Corpos d'água & 2 & $<1$ \\
\hline Floresta 2 ${ }^{\circ}$ est. inicial & 2 & 36 \\
\hline Floresta 2 ${ }^{\circ}$ est. médio & 1 & 15 \\
\hline Floresta 2 ${ }^{\circ}$ est. avançado & 1 & 2 \\
\hline Núcleo rural/Construções & 3 & 1 \\
\hline Pastagem & 4 & 23 \\
\hline Área alagável/Charco & 3 & 7 \\
\hline Pasto sujo/Campo & 3 & 8 \\
\hline Solo exposto/Área degradada & 5 & 1 \\
\hline
\end{tabular}

apoio sobre a imagem de satélite Ikonos (Spörl, 2007; Crepani et al., 2001).

A integração dos critérios foi realizada pela soma simples dos valores associados aos grids dos PI intermediários (intensidade pluviométrica, declividade do terreno, classes pedológicas e formas de uso e cobertura da terra). Esses PI foram convertidos do formato vetorial para modelo raster (matricial). Esse formato consiste em uma grade regular, representada por uma malha quadriculada, composta por células de dimensão espacial e valor atribuído definidos (Silveira \& Oka-Fiori, 2007). 


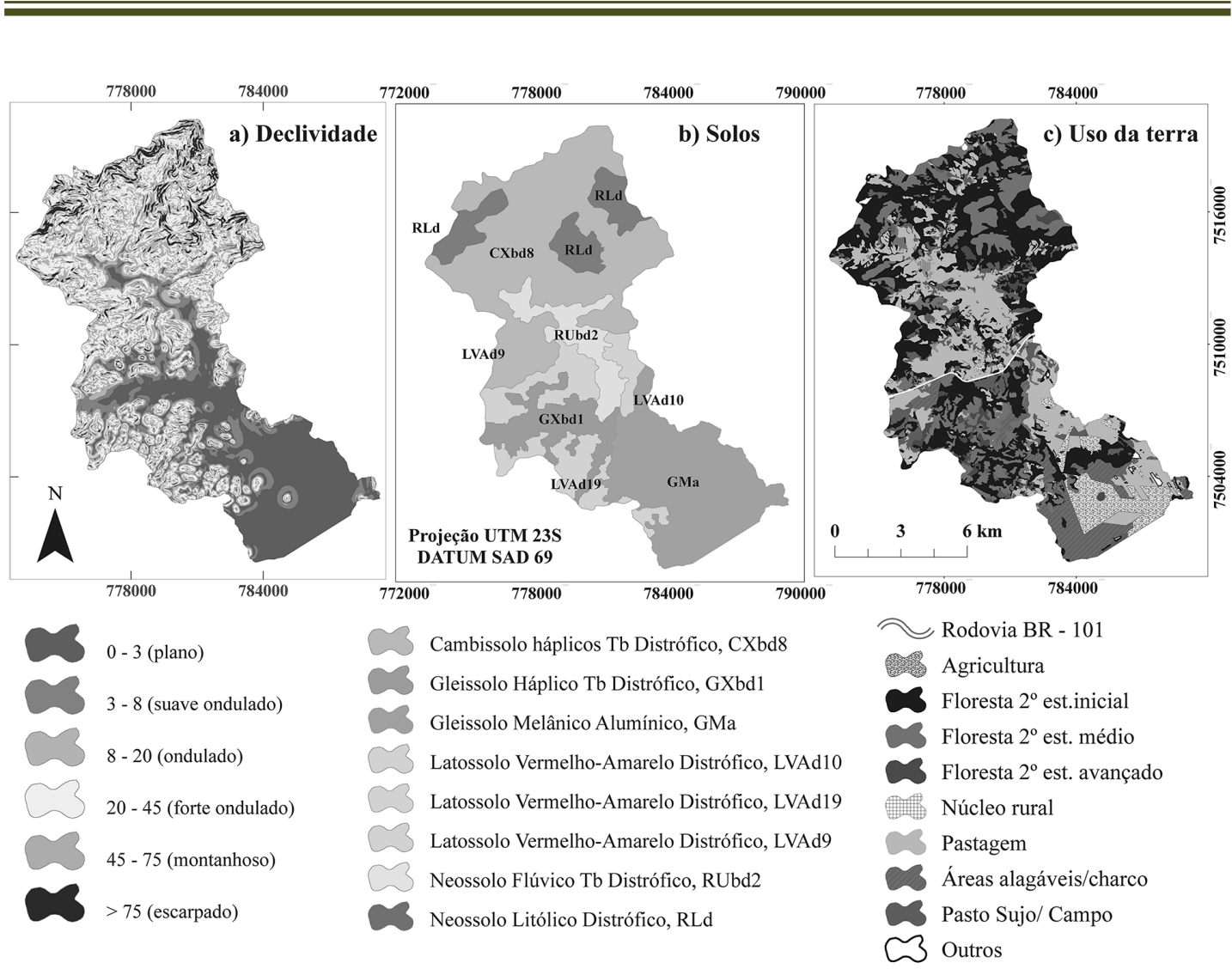

Figura 2. Classificação dos atributos do terreno: (a) Declividade; (b) Tipos de solo; (c) Uso e cobertura da terra. Figure 2. Classification of terrain attributes: (a) slope; (b) soil classes; (c) land use.

\subsection{Mapeamento da fragilidade ambiental}

O procedimento para o diagnóstico e mapeamento da fragilidade ambiental consistiu em uma análise multicriterial que teve como base as metodologias propostas por Ross (1994), Crepani et al. (2001) e Jain \& Goel (2002). A aplicação do método se baseou na integração de mapas de vulnerabilidade associada aos atributos de terreno (precipitação, declividade, solos, cobertura vegetal, uso e cobertura da terra). A partir da interpretação de mapas temáticos e consultas a especialistas (Galiana-Martín \& Karlsson, 2012) foram definidos critérios para a classificação e a hierarquização dos planos de informação (PI) e do grau de fragilidade ambiental, de acordo com a vulnerabilidade de cada área a sofrer processos de degradação do solo por erosão. O método proposto tende a realizar um diagnóstico do ambiente através dos atributos do terreno e suas contribuições nos diferentes graus de fragilidade ambiental. Cada variável foi representada hierarquicamente através de cinco classes de vulnerabilidade ambiental.
O Mapa de Fragilidade Potencial (MFP) foi elaborado por meio da sobreposição das informações de intensidade pluviométrica ( $\mathrm{mm} / \mathrm{ano}$ ), declividade do terreno (\%) e pedologia (unidades de mapeamento de solos). O Mapa de Fragilidade Emergente (MFE) foi obtido a partir da sobreposição do mapa resultante da Fragilidade Potencial (MFP) e do mapa temático com os valores estabelecidos para as diferentes classes de uso e ocupação da terra.

Foi realizada uma associação numérica entre os PI considerados para a definição das classes de fragilidade ambiental, potencial e emergente (Tabela 5). As classes de fragilidade foram definidas através do somatório dos valores inferidos para cada PI, dentro de intervalos predefinidos, considerando-se as possíveis combinações entre os fatores, referências literárias e opinião de especialistas. A partir dos PI intermediários, reclassificados de acordo com as pontuações atribuídas (intensidade pluviométrica, declividade do terreno, pedologia e uso/cobertura da terra), realizou-se álgebra de mapas para soma das pontuações e obtenção dos mapas de 
Tabela 5. Soma das pontuações e definição das classes de Fragilidade Potencial e Emergente.

Table 5. Sum of scores and description of the Potential and Emerging Fragility classes.

\begin{tabular}{|c|c|c|c|c|c|c|c|c|c|c|c|}
\hline \multicolumn{5}{|c|}{ Pontuações A + B + C } & $=$ & $\begin{array}{c}\text { Fragilidade } \\
\text { Potencial }\end{array}$ & + & D & $=$ & $\begin{array}{l}\text { Fragilidade } \\
\text { Emergente }\end{array}$ & $\begin{array}{c}\text { Descrição das } \\
\text { classes }\end{array}$ \\
\hline 1 & & 1 & & 1 & & 03 & & 1 & & 04 & Muito baixa \\
\hline 2 & & 2 & & 2 & & 04 a 06 & & 2 & & 05 a 08 & Baixa \\
\hline 3 & + & 3 & + & 3 & $=$ & 07 a 09 & + & 3 & $=$ & 09 a 12 & Média \\
\hline 4 & & 4 & & 4 & & 10 a 12 & & 4 & & 13 a 16 & Alta \\
\hline 5 & & 5 & & 5 & & 13 a 15 & & 5 & & 17 a 20 & Muito alta \\
\hline
\end{tabular}

Soma das pontuações: $\mathrm{A}$ = Pluviosidade; $\mathrm{B}=$ Declividade; $\mathrm{C}$ = Pedologia; $\mathrm{D}=$ Uso e ocupação da terra.

fragilidade (potencial e emergente). Nessa etapa, foi utilizada a Raster calculator/Map Algebra (ESRI, 2010).

Os mapas gerados foram validados através da sobreposição com os registros dos Processos Erosivos Aparentes (PEA). Os PEA foram identificados a partir da imagem do satélite Ikonos e posteriormente conferidos em campo com auxílio de GPS (Global Positioning System) quando acessíveis (mais de 75\% das ocorrências) durante o ano de 2010.

\section{RESULTADOS E DISCUSSÃO}

A localização e a topografia na região da Bacia do Rio Aldeia Velha favorecem a ocorrência de fortes chuvas orográficas, intensificadas no verão. Isso explica a atribuição de um valor constante para a fragilidade ambiental no que se refere ao fator pluviosidade - peso 4, vulnerabilidade Forte (Ross, 2012). Nesse sentido, o parâmetro utilizado pelo modelo considera a influência da ação do escoamento superficial das águas pluviais como uma superfície contínua. Os demais atributos do terreno, utilizados como critérios para o diagnóstico da fragilidade ambiental (potencial e emergente), apresentaram variabilidade espacial em diferentes níveis de fragilidade, dessa forma, são apresentados através de mapas temáticos (Figura 3).

De acordo com o mapa de declividade do terreno destaca-se o alto grau de vulnerabilidade ambiental associado às áreas montanhosas. No entanto, as condições limitantes à ocupação humana favorecem a manutenção da cobertura florestal nessas áreas, o que minimiza o risco à erosão relacionada aos fatores naturais na bacia em estudo (Fragilidade Potencial).

Em relação à pedologia, solos considerados jovens e pouco desenvolvidos apresentam grau máximo de vulnerabilidade ambiental como, por exemplo, os
Gleissolos e os Neossolos (Leme, 2007). Esse grau de vulnerabilidade atribuído pode ser explicado pelo baixo grau de desenvolvimento de processos pedogenéticos identificados pela distribuição dos horizontes/camadas. A hierarquização das classes pedológicas, de acordo com os pesos atribuídos por Crepani et al. (2001), apontou vulnerabilidade muito alta em áreas montanhosas e até mesmo em áreas de baixada. Nesse caso, associada à ocorrência de Gleissolos (valor 5) em planícies fluviais, com drenagem deficiente e baixa velocidade de infiltração da água, favorecendo o escoamento superficial. Os Neossolos Litólicos, que correspondem às áreas de maior declividade (escarpas associadas à Serra do Mar), por sua vez, propiciam a remoção de material do solo e inibem processos pedogenéticos.

Cambissolos Háplicos predominam nas áreas montanhosas, também propícias à ocorrência de processos erosivos pela ação do escoamento superficial, que corresponde à sua classificação como de forte vulnerabilidade aos agentes erosivos. Neste estudo, apenas nas unidades de mapeamento onde predominam os Latossolos foi atribuído valor igual a 1, que representa vulnerabilidade muito baixa à ação de processos erosivos.

O tipo de uso da terra influencia diretamente a vulnerabilidade do terreno à ação de agentes erosivos - a erodibilidade do solo depende diretamente da capacidade de infiltração da água (Jain \& Goel, 2002). Neste trabalho, os efeitos dos diferentes tipos de uso e cobertura da terra para estabilidade dos ambientes foram representados por três classes de fragilidade: Baixa, Alta e Média (36\%, 30\% e 17\% da área total da bacia, respectivamente).

A integração dos mapas de fragilidade por unidades de mapeamento, complementada pela consulta a especialistas para as pontuações aferidas, possibilitaram a construção da matriz de fragilidade e a interpretação 

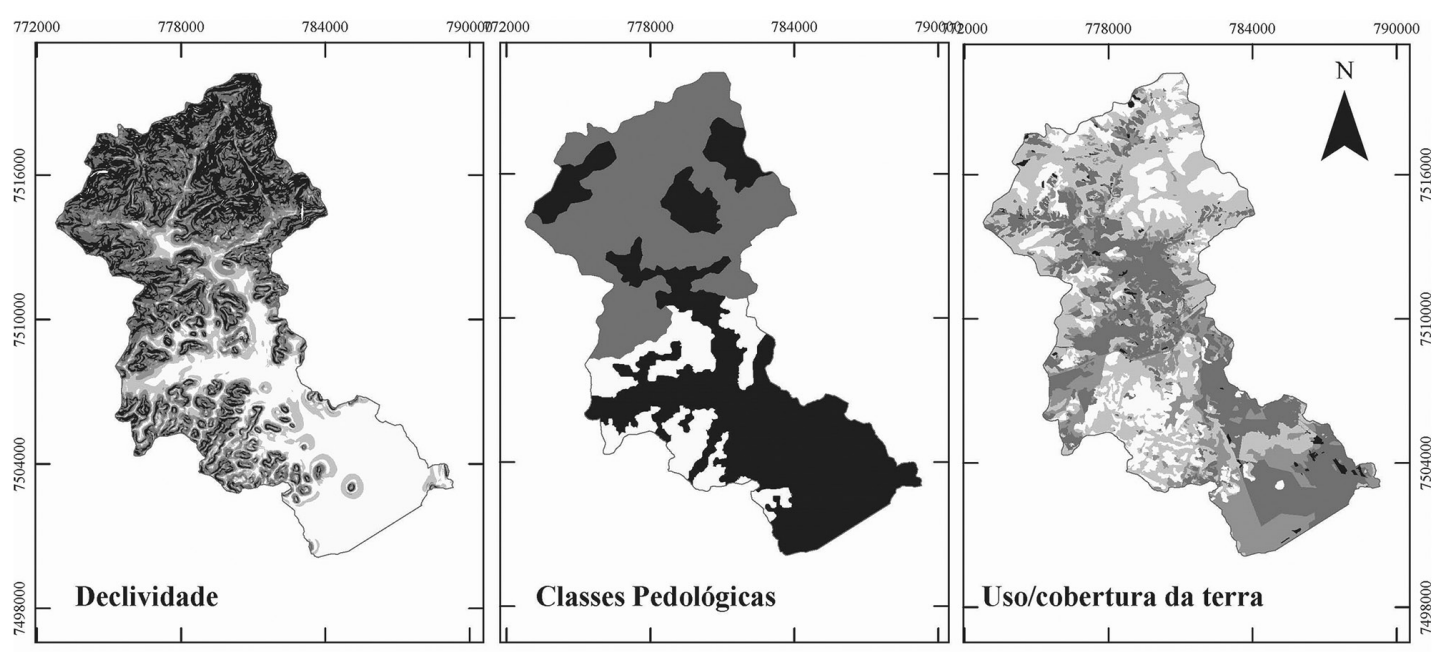

Fragilidade Ambiental

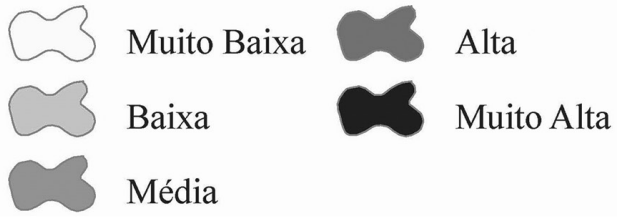

\section{Projeção UTM 23S - DATUM SAD 69}

Fontes: Valores de vulnerabilidade atribuídos às classes de declividade do terreno, classes pedológicas e uso/ cobertura da terra na bacia do rio Aldeia Velha, RJ.

Figura 3. Classificação da fragilidade ambiental relacionada aos atributos do terreno (declividade, pedologia e uso e cobertura da terra).

Figure 3. Classification of environmental fragility of terrain atributes (slope, pedology, and land use).

dos resultados. Através da sobreposição dos subprodutos foi possível verificar casualidade no fato de algumas unidades serem mais sujeitas à erosão do que outras, mesmo sob condições ambientais muito semelhantes.

A Bacia do Rio Aldeia Velha, através da integração dos atributos do terreno e uso da terra, em relação a seus efeitos sobre a estabilidade dos ambientes, foi majoritariamente representada pela classe de fragilidade alta (potencial e emergente), registrada em áreas agrícolas e de pastagem na bacia, localizadas principalmente na região das baixadas aluviais (Figura 4).

O MFE demonstra que a cobertura florestal é capaz de oferecer proteção ao solo, minimizar a intensidade e as consequências dos processos erosivos e, consequentemente, reduzir a fragilidade em determinados locais os quais, pela Fragilidade Potencial (MFP), seriam considerados de alta fragilidade. Em ambos os modelos gerados (MFE e MFP), os graus de fragilidade baixa e média predominaram em áreas caracterizadas por declividade inferior a $45 \%$ e pela ocorrência de Latossolos, localizadas em parte dos setores central e sudoeste da bacia, inclusive na área ocupada pela Rebio
Poço das Antas. A Tabela 6 apresenta a quantificação relativa em área, conforme o intervalo predefinido para o diagnóstico da fragilidade potencial (MFP) e emergente (MFE).

Analisando-se a distribuição relativa da área ocupada pelos diferentes graus de fragilidade foi possível constatar que, de uma forma geral, a bacia hidrográfica apresenta fragilidade potencial (MFP) alta e muito alta, que ocupam $87 \%$ da bacia; enquanto que no Mapa de Fragilidade Emergente (MFE) predominou a classe fragilidade alta, ocupando $68 \%$ da bacia, sendo a classe de fragilidade muito alta correspondente a $10 \%$ da área da bacia.

O modelo apresentado para fragilidade emergente, com o objetivo de subsidiar o planejamento de uso e o controle da erosão do solo na Bacia do Rio Aldeia Velha, indicou áreas de fragilidade muito alta em locais com declividade superior a $100 \%$ ou $45^{\circ}$ na linha de maior declive e nos terços superiores de morros e montanhas. Tais áreas são consideradas como prioritárias para a preservação das funções ecossistêmicas e são classificadas como Áreas de Preservação Permanente, 


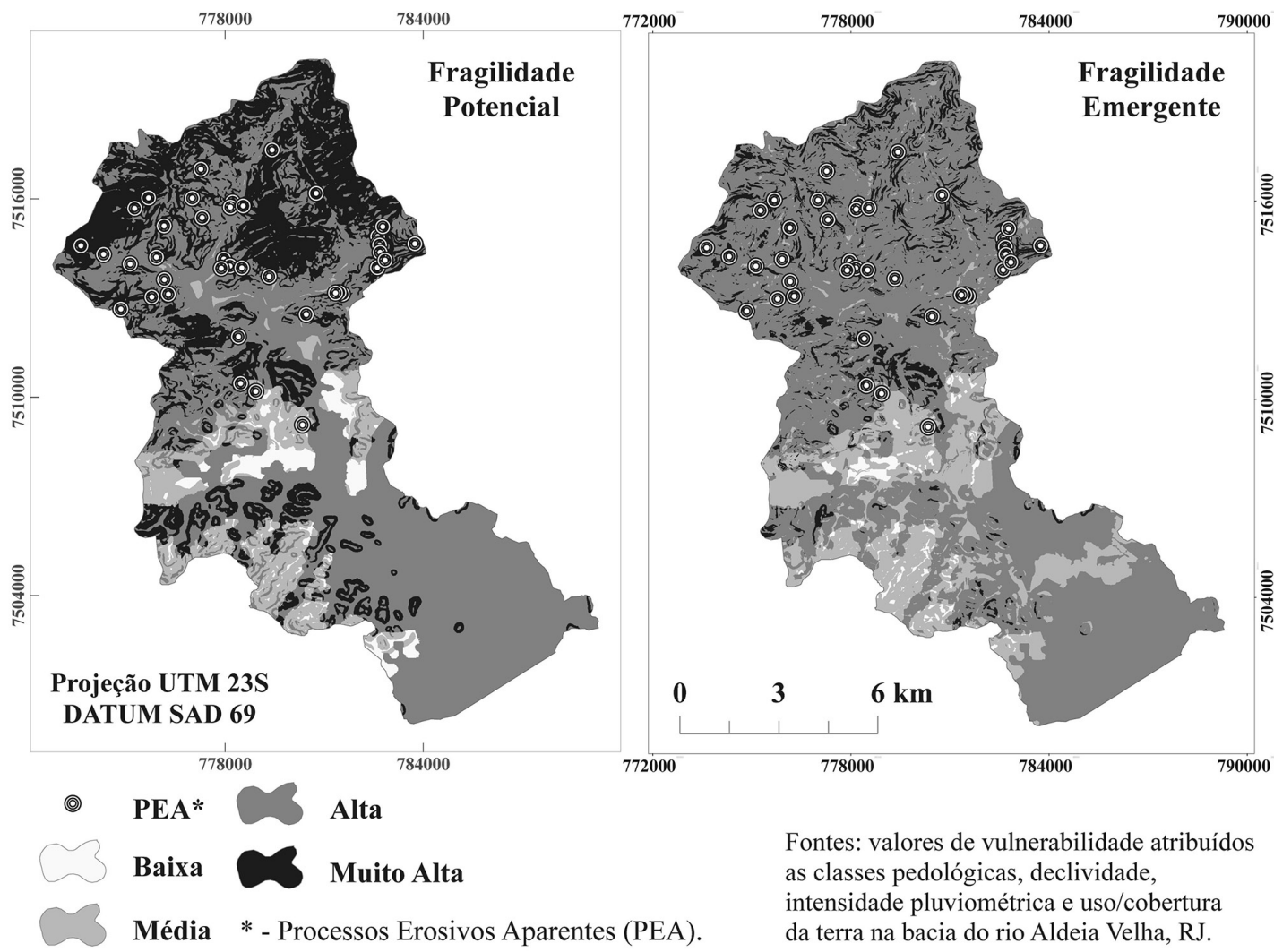

Figura 4. Mapas de Fragilidade Ambiental Potencial e Emergente na bacia hidrográfica. Figure 4. Map of Potential and Emerging Environmental Fragility in the watershed.

Tabela 6. Áreas das classes de fragilidade ambiental: Potencial e Emergente (FP e FE).

Table 6. Areas of fragility classes: Potential and Emergent (PF and EF).

\begin{tabular}{|c|c|c|c|c|}
\hline \multirow{2}{*}{ Classes } & \multicolumn{2}{|c|}{ FP } & \multicolumn{2}{|c|}{ FE } \\
\hline & Intervalo valores ${ }^{\star}$ & Área (\%) & Intervalo valores ${ }^{\star}$ & Área (\%) \\
\hline Baixa & 06 & 3 & 07,08 & 5 \\
\hline Média & $07,08,09$ & 10 & $09,10,11,12$ & 17 \\
\hline Alta & $10,11,12$ & 57 & $13,14,15,16$ & 68 \\
\hline Muito alta & $13,14,15$ & 30 & $17,18,19,20$ & 10 \\
\hline Total & & 100 & & 100 \\
\hline
\end{tabular}

${ }^{*}$ Intervalos considerados para as pontuações e descrição das classes nos Mapas de Fragilidade Potencial (MFP) e Emergente (MFE).

conforme dispõe o Código Florestal (Brasil, 2012). Relacionando-se o MFP com o MFE é possível constatar a função estratégica da cobertura florestal na proteção do solo e na contenção de processos erosivos.

Integrando-se o Mapa de Fragilidade Potencial (MFP) e o mapa de uso e ocupação da terra, o Mapa de Fragilidade Emergente (MFE) oferece melhor validação dos PEA (Processos Erosivos Aparentes), pois revela a influência das atividades antrópicas na estabilidade do ambiente, destacando a contribuição da cobertura florestal que oferece proteção às áreas de alto potencial natural à erosão, seja pelo fator declividade, seja pelo tipo de solo. Sendo assim, o mapeamento dos PEA confirmou a coerência da distribuição espacial do gradiente fragilidade ambiental produzido nesta pesquisa em função das intervenções antrópicas na paisagem.

A Tabela 7 apresenta a distribuição relativa dos processos erosivos aparentes em função das classes de fragilidade ambiental. 
Tabela 7. Número de ocorrências e distribuição relativa dos PEA (Processos Erosivos Aparentes) em função das classes de fragilidade ambiental.

Table 7. Number of occurrences and relative distribution on the classes of environmental fragility of apparent erosion processes (PEA).

\begin{tabular}{ccccc} 
& \multicolumn{4}{c}{ Processos Erosivos Aparentes (PEA) } \\
\cline { 2 - 5 } & \multicolumn{2}{c}{ Fragilidade Potencial } & \multicolumn{2}{c}{ Fragilidade Emergente } \\
\cline { 2 - 5 } Classes & Absoluto & Relativo (\%) & Absoluto & Relativo (\%) \\
Baixa & --- & -- & --- & 5 \\
Média & 2 & 5 & 2 & 27,5 \\
Alta & 20 & 50 & 11 & 67,5 \\
\hline Muito alta & 18 & 45 & 27 & - \\
\hline
\end{tabular}

No total, foram identificados 40 pontos que retratam cicatrizes de deslizamentos em encostas. No MFP, observa-se que apenas 2 PEA localizam-se em áreas de fragilidade média; 20 localizam-se em áreas de fragilidade alta; e 18, em áreas classificadas como de fragilidade muito alta. A maioria dos PEA foi registrada em encostas de alta declividade e solo pouco desenvolvidos (Neossolos), os quais combinados com a forte intensidade das chuvas determinam as maiores manchas de fragilidade muito alta, confirmando a alta susceptibilidade do ambiente natural e a pouca estabilidade das vertentes ante a morfodinâmica da área analisada.

Em relação ao MFE, 2 pontos de PEA localizam-se em área de fragilidade média; 11 pontos localizam-se em áreas classificadas como fragilidade alta; e 27, em áreas de fragilidade muito alta. O registro desses fenômenos sugere que a representação da Fragilidade Emergente é capaz de registrar com boa precisão os locais de alta susceptibilidade à erosão na área estudada. Apesar de as áreas de fragilidade muito alta terem diminuído no MFE, resultado da estabilidade proporcionada pela cobertura florestal, a maioria dos PEA concentra-se nessa classe de fragilidade, confirmando a coerência das classificações nesse mapa síntese.

Foi possível observar a influência que a perda de cobertura florestal exerceu sobre a estabilidade do solo; PEA classificados como de fragilidade alta no MFP foram convertidos para de fragilidade muito alta no MFE. Esse fato pode resultar de formas de ocupação ou manejo inadequadas em terrenos mais suscetíveis à erosão, como áreas de maior declividade. Da mesma forma como a cobertura florestal oferece maior proteção em relação aos processos erosivos, a atividade antrópica é capaz de potencializá-los. A cobertura florestal é capaz de proteger o solo, favorecendo a capacidade de infiltração da água e a redução do escoamento superficial, além de auxiliar na manutenção de diversas relações ecológicas, representando um agente de equilíbrio no ambiente e contribuindo para diminuir a susceptibilidade do terreno à erosão (Kawakubo et al., 2005).

Em geral, 45\% e 67\% dos PEA localizam-se em áreas de fragilidade muito alta no Mapa de Fragilidade Potencial (MFP) e no Mapa de Fragilidade Emergente (MFE), respectivamente. Entre os PEA identificados, três pontos classificados como de fragilidade potencial muito alta (MFP) descenderam para a classe alta quando considerada a cobertura florestal no MFE. Dessa forma, recomendamos que novas formas de ordenamento e manejo conservacionistas sejam praticadas, sobretudo em áreas de exploração agropecuária em que predominam valores de fragilidade muito alta. $\mathrm{Na}$ área de estudo, as áreas de relevo montanhoso foram as que apresentaram os mais altos valores de fragilidade ambiental, embora com cobertura florestal. Fatores como tipo de solo e declividade são decisivos para se impor limitação às formas de ocupação, recomendadas para preservação da fauna e flora nessas áreas. Essas precauções são fundamentais para a manutenção de ecossistemas locais e controle dos processos erosivos, contribuindo para garantir a qualidade dos recursos hídricos e o abastecimento de água na região.

\section{CONCLUSÃO}

Considerando-se o modelo produzido, a área em estudo foi geralmente diagnosticada como de alta fragilidade ambiental. Isso indica a susceptibilidade da ocorrência de processos erosivos, considerando-se as características topográficas e naturais, assim como as intervenções antrópicas no meio ambiente. A representação da fragilidade ambiental como produto do conjunto das 
interpretações dos diferentes Planos de Informação (PI) intermediários conferiu um caráter holístico à análise e interpretação das condições ambientais relacionadas aos fatores limitantes ao uso e ocupação das terras, processo útil ao planejamento territorial e de réplica relativamente acessível, devido à disponibilidade dos dados oficiais e às técnicas de análise. Os fatores avaliados na análise são capazes de exercer grande influência no desenvolvimento de processos sociais e ecológicos. O produto dessas interações complexas pode contribuir para o equilíbrio ambiental, trazendo benefícios econômicos e ecológicos.

O Mapa de Fragilidade Emergente (MFE) destaca o papel da cobertura florestal para a estabilidade no ambiente, reduzindo a área de fragilidade muito alta. Dessa forma, foram identificadas áreas prioritárias para a preservação e conservação ambiental, de grande susceptibilidade natural à erosão, vinculadas aos declives da região montanhosa e aos solos pouco desenvolvidos na área de estudo. O MFE representou as áreas mais propensas à ocorrência de Processos Erosivos Aparentes (PEA) e pode ser um instrumento importante para o planejamento territorial em bacias hidrográficas.

Os atributos considerados para o diagnóstico foram sensíveis às características naturais do ambiente. Os PEA localizaram-se geralmente em áreas identificadas como de fragilidade alta e muito alta nos produtos cartográficos finais. A variável uso/cobertura da terra foi determinante para validação desses fenômenos no MFE, demonstrando a influência que a forma de ocupação exerce sobre a estabilidade do terreno. A análise permitiu a interação entre dados de natureza distinta, possibilitando o diagnóstico da fragilidade ambiental a partir da interpretação dos diversos Planos de Informação (PI).

O método empregado é promissor como subsídio à tomada de decisões no que tange a intervenções na paisagem, gestão ambiental e planejamento territorial, como para a elaboração de programas de preservação de áreas florestais e de contenção de processos erosivos, principalmente por prefeituras municipais e organizações da sociedade civil (ONGs). A identificação de áreas de risco, considerando-se a vulnerabilidade natural do ambiente, juntamente com as validações das classificações da terra são essenciais para o monitoramento e a elaboração de Planos Diretores e de Manejo e para projetos conservacionistas. Os produtos cartográficos finais foram capazes de traduzir a realidade ambiental de maneira simples e de fácil compreensão, além de serem passíveis de atualização em função da base de dados de entrada nos modelos como, por exemplo, mapas de uso e cobertura mais recentes.

Nosso modelo considerou a pontuação atribuída às classes como qualitativa, na medida em que aos atributos foi conferido o mesmo peso na qualidade ambiental. Esse procedimento para análise pode representar uma limitação em determinadas regiões, nas quais alguns dos fatores considerados possam representar uma maior ou menor contribuição para a qualidade e a fragilidade ambiental, sugerindo a necessidade de ponderação dos critérios, além da pontuação hierárquica. Entretanto, estudos dessa natureza são capazes de contribuir para a gestão de bacias hidrográficas através da representação da fragilidade das terras e do potencial natural para erodibilidade, uso agrícola e florestal. O aprimoramento dessas ferramentas possibilita um diagnóstico cada vez mais eficiente da fragilidade dos ambientes, através do zoneamento das áreas de expansão urbana e recuperação/preservação de áreas prioritárias.

\section{AGRADECIMENTOS}

Agradecemos ao Programa de Pós-Graduação em Ciências Ambientais e Florestais (PPGCAF/UFRRJ), à equipe da Associação Mico-Leão-Dourado (AMLD), da REBIO Poço das Antas e da Prefeitura Municipal de Silva Jardim (PMSJ-RJ).

\section{STATUS DA SUBMISSÃO}

Recebido: 6 ago., 2014

Aceito: 8 nov., 2015

\section{AUTOR(ES) PARA CORRESPONDÊNCIA}

\section{Ivana Cola Valle}

Departamento de Ciências Biológicas, Universidade Estadual de Santa Cruz - UESC, Rodovia Ilhéus/Itabuna, Km 16, CEP 45662-000, Ilhéus, BA, Brasil e-mail: ivana_geouff@yahoo.com.br 


\section{REFERENNCIAS}

Bojórquez-Tapia LA, Cruz-Bello GM, Luna-González L. Connotative land degradation mapping: a knowledge-based approach to land degradation assessment. Environmental Modelling \& Software 2013; 40: 51-64. http://dx.doi. org/10.1016/j.envsoft.2012.07.009.

Brasil. Lei $n^{\circ} 12.651$ de 25 de maio de 2012. Novo Código Florestal Brasileiro. Diário Oficial da República Federativa do Brasil, Brasília, DF (2012 maio 25) [citado em 2010 set 01]. Disponível em: http://www.planalto.gov.br/ ccivil_03/_Ato2011-2014/2012/Lei/L12651.htm

Brasil. Ministério do Meio Ambiente. Revisão do Plano de Manejo da Reserva Biológica Poço das Antas. Brasília: MMA; 2005.

Burrough PA, McDonnell RA. Creating continuous surfaces from point data: principles of geographic information systems. Oxford: University Press; 1998.

Cardinali M, Reichenbach P, Guzzetti F, Ardizzone F, Antonini G, Galli M et al. A geomorphological approach to the estimation of landslide hazards and risks in Umbria, Central Italy. Natural Hazards and Earth System Sciences 2002; 2(1/2): 57-72. http://dx.doi.org/10.5194/ nhess-2-57-2002.

Carvalho AD FO, Lumbreras JF, Wittern KP, Lemos AL, Santos RD, Calderano B FO et al. Mapa de reconhecimento de baixa intensidade dos solos do estado do Rio de Janeiro [map]. Rio de Janeiro: Embrapa Solos; 2003. Mapa Colorido; Escala 1: 250.000.

Crepani E, Medeiros JS, Azevedo LG, Duarte V, Hernandez P, Florenzano T et al. Sensoriamento remoto e geoprocessamento aplicados ao zoneamento ecológicoeconômico e ao ordenamento territorial. São José dos Campos: INPE; 2001.

Embrapa. Sistema brasileiro de classificação de solos. Rio de Janeiro: EMBRAPA; 2006.

Environmental Systems Research Institute - ESRI. Software ArcGIS desktop: version 10. Redlands: Environmental Systems Research Institute; 2010.

Francisco CES, Coelho RM, Torres RB, Adami SF. Análise Multicriterial na seleção de bacia hidrográfica para recuperação ambiental. Ciência Florestal 2008; 18(1): 1-13.

Franco GB, Betim LS, Marques EAG, Gomes RL, Chagas CS. Relação qualidade da água e fragilidade ambiental da Bacia do Rio Almada, Bahia. Brazilian Journal of Geology 2013; 42: 114-127.

Galiana-Martín L, Karlsson O. Development of a methodology for the assessment of vulnerability related to wildland fires using a multi-criteria evaluation. Geographical Research 2012; 50(3): 304-319. http://dx.doi.org/10.1111/j.17455871.2011.00718.x.
Instituto Brasileiro de Geografia e Estatística - IBGE. Manual técnico de pedologia. Rio de Janeiro: IBGE; 2007. 316 p. Manuais Técnicos em Geociências n. 4.

Instituto Brasileiro de Geografia e Estatística - IBGE. Cartas de Quartéis (SF-23-Z-B-III-3-SE), Casimiro de Abreu (SF-23-Z-B-III-4-SO), Silva Jardim (SF-23-Z-BVI-1-NE) e Morro de São João (SF-23-Z-B-VI-2-NO). Bases cartográficas em ambiente digital (formato dgn, pdfe tif), na escala 1:50.000, agrupadas por categorias de informação: hipsometria, hidrografia e limites políticos. Rio de Janeiro: IBGE; 2010. [citado em 2010 set 01]. Disponível em: www.ibge.gov.br.

Jain SK, Goel MK. Assessing the vulnerability to soil erosion of the Ukai Dam catchments using remote sensing and GIS. Hydrological Sciences Journal 2002; 47(1): 31-40. http://dx.doi.org/10.1080/02626660209492905.

Kappes MS, Papathoma-köhle M, Keiler M. Assessing physical vulnerability for multi-hazards using an indicatorbased methodology. Applied Geography (Sevenoaks, England) 2012; 32(2): 577-590. http://dx.doi.org/10.1016/j. apgeog.2011.07.002.

Kawakubo FS, Morato RG, Campos KC, Luchiari A, Ross JLS. Caracterização empírica da fragilidade ambiental utilizando geoprocessamento. In: Anais XII Simpósio Brasileiro de Sensoriamento Remoto; 2005; São José dos Campos. Goiânia: INPE; 2005. p. 2203-2210.

Köppen W. Climatologia: con un estudio de los climas de la tierra. Ciudad de México: Fondo de Cultura Econômica; 1948; 479p.

Leme SM. Relevo, processos geoecológicos e sócio/ reprodutores e a fragilidade ambiental da bacia do ribeirão Piracicamirim/ SP [tese]. São Paulo: Faculdade de Filosofia, Letras e Ciências Humanas, Universidade de São Paulo; 2007.

Martín-Duque JF, García JC, Urquí LC. Geoheritage information for geoconservation and geotourism through the categorization of landforms in a Karstic Landscape: a case study from Covalagua and Las Tuerces (Palencia, Spain). Geoheritage 2012; 4(1-2): 93-108. http://dx.doi. org/10.1007/s12371-012-0056-2.

Montebeller CA, Ceddia MB, Carvalho DFD, Vieira SR, Franco EM. Variabilidade espacial do potencial erosivo das chuvas no Estado do Rio de Janeiro. Engenharia Agrícola 2007; 27(2): 426-435.

Ratcliffe DA. Criteria for the selection of nature reserves. Advancement of Sciences 1971; 27: 294-296.

Rodrigues JBT, Zimback CRL, Piroli EL. Utilização de Sistema de Informação Geográfica na Avaliação do Uso da Terra em Botucatu (SP). Revista Brasileira de Ciência do Solo 2001; 25(3): 675-681. http://dx.doi.org/10.1590/ S0100-06832001000300016.

Ross JLS. Análise empírica da fragilidade dos ambientes naturais e antropizados. Revista do Departamento de Geografia 1994; 8: 63-74. 
Ross JLS. Landforms and environmental planning: Potentialities and Fragilities. Revista do Departamento de Geografia 2012; 38-51.

Silveira ACT, Oka-Fiori C. Análise empírica da fragilidade potencial e emergente na Bacia do Rio Cubatãozinho, Estado do Paraná. Caminhos da Geografia 2007; 8: 1-17.

Spörl C, Ross JLS. Análise da fragilidade ambiental relevosolo com aplicação de três modelos. GEOUSP - Espaço e Tempo 2004; 15: 39-49.

Spörl C. Metodologia para elaboração de modelos de fragilidade ambiental utilizando redes neurais [tese]. São Paulo: Faculdade de Filosofia, Letras e Ciências Humanas, Universidade de São Paulo; 2007.
Tomczyk AM. A GIS assessment and modelling of environmental sensitivity of recreational trails: The case of Gorce National Park, Poland. Applied Geography (Sevenoaks, England) 2011;31(1): 339-351. http://dx.doi. org/10.1016/j.apgeog.2010.07.006.

Tricart J. Ecodinâmica. Rio de Janeiro: IBGE; 1977. Série recursos naturais e meio ambiente.

Valle IC, Buss DF, Baptista DF. The influence of connectivity in forest patches, and riparian vegetation width on stream macroinvertebrate fauna. Brazilian Journal of Biology = Revista Brasileira de Biologia 2013; 73(2): 231-238. http://dx.doi.org/10.1590/S1519-69842013000200002. PMid:23917549. 